\title{
Application of Yangshao Decorative Pattern Elements in Font Design of Chinese Characters
}

\author{
Juanjuan $\mathrm{Su}$ \\ Eastern International Art College \\ Zhengzhou University of Light Industry \\ Zhengzhou, China
}

\begin{abstract}
With the advance of globalization, China's economy develops rapidly and China's international status has been promoted constantly. Chinese culture has drawn world attention. More and more Chinese modern designs are recognized in the world, which is due to the reasonable and effective application of traditional patterns. Chinese Yangshao colored pottery patterns contain the most primitive spirits of our ancient nation in creation and aesthetics. In the course of history, Chinese traditional patterns have expressed good implication with patterning, and combined an advantageous design concept in modern design, eliminating the boundaries between elegance and popularity, tradition and modern. This paper hopes to combine graphics morphing and font design together by the analysis of the characteristics of Yangshao colored pottery patterns.
\end{abstract}

Keywords—colored pottery; pattern; font design

\section{THE HistoricAl DEVELOPMENT OF CHINESE YANGSHAO COLORED POTTERY PATTERNS}

Yangshao culture was first discovered in Yangshao Village, Mianchi County, Henan in 1921, hence its name. Its distribution range is roughly in Guanzhong Area of Shaanxi Province, most areas of Henan Province, the southern part of Shanxi and Hebei, as far as the junction of Gansu and Qinghai, Hetao Area, the northern part of Hebei, the northwestern part of Hubei and other places, which is about $7000 \sim 5000$ years ago.

The historical course of Yangshao cultural pattern is divided into three stages:

The first stage: ancient human pictographic noting primitive drawings were graphical symbols of primitive man. It is the primitive form of graphics, and also the text prototype.

The second stage: the pictorial symbols were evolved into characters. The pictorial symbols are different from noting drawings. They are more abstract and simplified. The noting drawings were simplified in practice on and on, and then formed graphical characters.

The third stage: the generation of characters had brought the development of graphics. The visual delivery form of character made human's communication closer. It can convey complex information and be easily understood, so this graphical form was valued and used by human.
Similar to the historical course of graphics, the academic circles divided the development stage of Yangshao culture into three stages, early stage, middle stage and late stage, according to the changes of sharp bottom vase.

\section{A. The Early Stage of Yangshao Culture (About $7000 \sim 6000$ Years Ago)}

Decorative features: most are rope patterns, as well as lined pattern and string pattern. Yangshao colored pottery has made brilliant achievements. The early Yangshao colored pottery bid farewell to the naive childhood, and began to go to maturity gradually. The artistic creativity of ancestors had a great progress, compared with Dadiwan culture. They had designed a variety of patterns, and their work level was highly improved in quantity and quality. According to the statistics of pottery pieces in the second stage of Dadiwan culture, colored pottery accounts for about $18.6 \%$. Colored pottery discovered in the second stage of Xishanping accounts for about $13.3 \%$. And the proportion of colored pottery gradually increased from early stage to late stage.

Fish pattern is the most common pattern of colored pottery in the early stage of Yangshao culture, and it has continued to be used till the middle stage of Yangshao culture. It lasted for a thousand years. After the middle stage of Yangshao culture, fish pattern appeared sporadically or occasionally, and it was no longer as the theme pattern. In most of the time they were drawn on the middle upper outer bellies of basin-shaped wares. In the rare case it was used on surrounding-bottom pots, bottleshaped utensils and their covers. The fish patterns on basinshaped ware are structured and well-aligned, which is very fixed. Generally there are two fishes in a round of the ware. The two fishes are arranged head to tail. The head is towards right, and the tail towards left. Sometimes, the blank space between the two fishes is filled with a small fish pattern or other patterns, which has made up the defects in the rendering process, and made the entire pattern more well-balanced and vivid. Sporadic fish patterns would appear on other wares, such as the cover of bottle-shaped head ware discovered in Wangjia Yinwa. Although few in number, but they are charismatic, revealing the outstanding skills of prehistoric painters. In the Yangshao cultural relics of the Weihe River Basin, we unearthed a large number of colored potteries with fish pattern. Among them Qin'an Dadiwan has the most abundant. In this relics unearthed more than 30 pieces of complete and recoverable basins with fish pattern, and a 
thousand fish pattern pieces. What's more, these fish patterns constitute a development sequence from early stage to late stage. According to their located stratum and overlying relationships, we generally grasped the development law of fish pattern. Therefore, it can be the scaleplate for archaeological research to dividing the history into stages.

Dadiwan fish patterns are a side view of fish, except the painting of pectoral fin. The pectoral fins should be on both sides of the body, and only one side of the pectoral fin can be observed. However, Dadiwan ancestors changed it and made them longitudinally symmetrical. No matter how fish patterns change, the painting of tail fin is always straight. Analyzing from the painting of fish fins, the fish they painted belongs to freshwater fish. Obviously, located in the inland areas, Yangshao ancestors could only describe fish they were familiar in their daily life. According to different characteristics of ware shapes and decorative patterns, we will divide the fish patterns of Dadiwan Yangshao culture into five developmental stages.

The first stage: the fish pattern is of strongly realistic style. In this period, all pottery basins had rounded bottom and wideflared mouth. Clay for its mouth edge is thicken and overlapped, so it is called overlapping lip basin. The fish pattern is lively and vivid, especially the oval eye and eyeball in the upper of orbit. They made the fish lifelike. With incomplete fins, no back and pelvic fins, the fish has mouth, eye, gills, body and tail, which is very clearly.

The second stage: they are still overlapping lip basin. Fish head became longer and patterned. With complicated and fickle painting techniques, the detail of head makes us obscure. Mostly use triangle pattern composed by straight line and arc line to fill fish head. Fish body became more perfect. Most of fish patterns have complete chest, belly, back and tail fin. For the cause of this change and the specific meaning of various lines on head, there is no academic consensus. But it is certain that the abstract painting has been introduced into fish pattern.

The third stage: fish patterns were still used in overlapping lip basin, but the caliber of the basin gradually increased, the belly became shallow. The most significant changes and features in this stage is that the head part became extremely simple and abstract. It is composed of two curved lines in an up-down position. The head is flat and long. The painting technique of fish fins changed from oblique triangle pattern into straight triangle pattern. The painting technique became more structured.

The fourth stage: the ware shape with fish pattern changed from overlapping lip basin into edge rolling basin. The edge rolling basin still has round bottom, but its mouth edge rolls outward, and so it is called edge rolling basin. It was one of potteries people often used in daily life. They are often unearthed in the housing site and pit. At this time the fish pattern is simplified into a very stable pattern. Chest, belly and anal fin integrate into one, sitting opposite to elongated dorsal fins in an up-down position. In front of tail fin appears a cutoff line. The upper part and lower part of fish are completely symmetrical. And lines become smooth and graceful. In F1 housing site, unearthed two large fish pattern basin with a caliber more than $50 \mathrm{~cm}$. The two potteries often are as colored pottery boutique and sent abroad for exhibition.

The fifth stage: still used edge rolling basin. The shape of basin mouth changed from wide-flared mouth into straight mouth. The edge rolls curlier. This stage has stepped into the middle Yangshao culture. Although we didn't find a complete ware of this stage in Dadiwan, we can speculate the recovered ware shape and patterns according to the residuals unearthed from the Dadiwan. By reference to similar wares of same stage unearthed from Gansu Heshui and Shaanxi Qishan Wangjiazui Ruins, we are sure that our speculation and recovery conform to the developmental law of ware shape and pattern decoration. Fish pattern is more simplified, and fish head is represented by a dot. Fish body is simplified into four arcs. Omit all upper and lower fins, and only keep exaggerated, stretched tail fin.

\section{B. The middle stage of Yangshao culture (about 6000 5500 years ago)}

It is a period of rapid development and expansion, which has a significant impact on our prehistoric culture. Just a few hundred years, the typical cultural factors, such as, curve leaf pattern, dot pattern, arc triangle pattern and double-lip sharpbottom vase, quickly were spread throughout the vast areas of northern China, and even to the Yangtze River.

"Huashan Rose" refers to a typical colored pottery pattern of middle stage in Guanzhong Area. The colored potteries with flower pattern are native-born and unique in all primitive cultures. Chinese ethnic group and the culture are undoubtedly native. This statement makes the archaeological circles very shocked. The origin of the name of China has no final conclusion yet, but Yangshao culture, colored pottery pattern decoration and its achievements and special constructions to Chinese culture should be fully affirmed and reexamined.

\section{The Late Stage of Yangshao Culture (About $5500 \sim 4900$ Years Ago)}

It is about $5500 \sim 4900$ years ago, which is the most glorious historical stage for prehistoric culture in Gansu.

The colored pottery of late stage is divided into two categories. The first category is still known as colored pottery we often see, uneasy to fall off. The second category is painted pottery which is painted after the firing. There were only a few before. But in this stage there were a lot of painted potteries.

In the period of Yangshao culture, the colored pottery art gradually went towards prosperity. All kinds of simple and elegant decorative patterns were born in this period. Among them human face pattern and fish pattern are the most typical representatives. Relatively speaking, the most frequently used pattern is fish pattern in the earliest. After studying on the pattern evolvement of colored pottery pattern in Yangshao culture, we found that the pattern evolved from realistic concrete into abstract pattern, with fish patterns as main clue. This process can be roughly divided into three stages. First is the realistic depicting stage; then is decorative realistic stage, which is to give object patterned processing; the last is the abstract stage, which gets rid of object shape completely and changes into geometric symbols, irrelevant to natural object. 


\section{THE FORMATION METHOdS AND STRUCTURE OF CHINESE CHARACTERS}

In terms of the formation methods of Chinese characters, there has been a saying of "six categories" since the Han Dynasty. The first is the pictographic method. In general, the formation of Chinese characters contains pictographic method, self-explanatory method, associative method and pictophonetic method. In ancient times, we have the saying of "six categories" in the formation of Chinese characters. In addition to above four methods, it also includes mutual-explanatory method and borrowing method. But strictly speaking, these two methods should belong to the methods of applying Chinese characters.

In addition, the formal structure of Chinese characters can be divided into four levels, Chinese characters, components and strokes, stroke shapes.

According to the number of components in Chinese character, Chinese characters can be divided into single character and compound character. The single characters are of single structure. The compound characters have the following 12 types of structures:

1. (left-right structure), such as, “伟”, “林”. 2. (upper-lower structure), such as, “志”, “苗”, “字”. 3. (left-middle-right structure), such as, “湖”, “谢”. 4. (upper-middle-lower structure), such as: “毫”, “䯽”. 5. Semi-enclosed structure: (1). (upper-right surrounding structure), such as, “句”, “司”. (2). (left-right surrounding structure), such as, “庙”, “尼”. (3). (left-lower surrounding structure), such as, “连”, “建”. (4). (upper three-side surrounding structure), such as, “闹”, “问”. (5). (lower three-side surrounding structure), such as, “函”, “画”. (6). (left three-side surrounding structure), such as, “匣”, “巨”. 6. (whole surround structure), such as, “团”, “图”. 7. (mosaic structure), such as, “爽”, “哥”,

The components of all Chinese characters can be classified into one of the above structures. Some people consider the structure of “品”, “晶”, “森” as “品”-shaped structure, and the structure of “㗊”-shaped Chinese characters as “田”-shaped structure.

\section{THE CHARACTERISTICS OF THE COMBINATION OF YANGSHAO COLORED POTTERY PATTERNS AND CHINESE CHARACTERS FONT DESIGN}

The representative Yangshao patterns include plant pattern, fish pattern and geometric pattern. Abstract and simplify these patterns and blend in strokes of Chinese characters, and design. Then substitute these designed strokes into the formation methods and structures of Chinese characters for verification, to achieve a sense of beauty after combination. In designing strokes, we should first analyze Chinese characters profoundly, and grasp the structure of strokes of Chinese characters. The combined Chinese characters shall be visually balanced. We should try our best to make designed strokes apply to both simple and complicated characters. After font design, we should choose a suitable expression form to reach the harmony and unity among all expression forms, and make the font overall beautiful.

\section{A. The Relationship between Pattern Content and the Font Design Style}

Character design means to carefully arrange characters overall according to the visual design law. Character design is a product of human's production and practice, which gradually matures along with the development of human civilization. Before carrying out character design, we should first have a general understanding on its history and evolvement.

Fish head became longer and patterned. With complicated and fickle painting techniques, the detail of head makes us obscure. Mostly use triangle pattern composed by straight line and arc line to fill fish head. Fish body became more perfect. Most of fish patterns have complete chest, belly, back and tail fin. The head part became extremely simple and abstract. It is composed of two curved lines in an up-down position. The head is flat and long. The painting technique of fish fins changed from oblique triangle pattern into straight triangle pattern. The painting technique became more structured. Fish pattern is more simplified, and fish head is represented by a dot. Fish body is simplified into four arcs. Omit all upper and lower fins, and only keep exaggerated, stretched tail fin.

In the combination of fish pattern and Chinese characters, we extracted fish head and two side of body to combine with characters. Fish pattern font design is diverse, lively and with a wide range of applications.

Most of patterns on colored potteries are geometric patterns, starting from broadband pattern. Bowls and pots of all kinds of cultural colored potteries have a broadband pattern outside mouth edge. It is the origin of colored pottery pattern, existing in all the development course of cultural colored potteries. It is mainly composed of long or short, thick or thin, curve or straight, transverse and vertical lines, crossed lines and dots.

In the combination of geometrical pattern and Chinese characters, we selected the most frequently used triangle and circle pattern for design. The font layering varies abundantly, and the font is balanced and full of sense of the times.

Plant pattern have two expression forms: one is radial spin flower pattern. Generally two-dimension series pattern encloses the body of ware. The other is leaf pattern. With simple leaf as motif, associate together and constitute a pattern. Common plant patterns are flower petal pattern, pod pattern, leaf pattern, flower pattern and so on.

In the combination of plant patterns and Chinese characters, with many arcs in plant patterns, the plant pattern font is mellow and full. The strokes vary abundantly, and the contrast of thickness and thinness is strong.

\section{B. The Expression Form of the Combination of Pattern and Font}

The decorative fonts combined with pattern and font design have uses exaggeration, light and shade, increase or decrease in strokes, decoration and other techniques. With rich imagination, designers reconstructed character pattern, which 
strengthens character features, and enriches the connotation of standard fonts. At the same time, it not only requires single character shape to be beautiful, but also the overall style harmonious and united, and to be with readable concept and content to facilitate the dissemination of information.

With creative ideas for font design, the next is to select adaptive materials and forms. According to different types of design, select adaptive materials. The selected materials are not necessarily expensive and luxury, for expensive materials may not adapt to the category of the book and reflect design quality. Cheep materials may not be unable to reach the ideal artistic effect. With harmonious and united materials and forms, even the cheap materials could become irreplaceable unique art language in design, leaving readers with a simple and highgrade impression.

In summary, with the advance of globalization, China's economy develops rapidly and China's international status has been promoted constantly. Chinese culture has drawn world attention. More and more Chinese modern designs are recognized in the world, which is due to the reasonable and effective application of traditional patterns. Chinese Yangshao colored pottery patterns contain the most primitive spirits of our ancient nation in creation and aesthetics. In font design of Chinese characters, the combination of Chinese traditional Yangshao patterns and font have expressed good implications, and combined an advantageous design concept in modern design, eliminating the boundaries between elegance and popularity, tradition and modern.

\section{REFERENCES}

[1] Li Xuewu. Chinese Primitive Colored Potteries. Jiangxi Fine Arts Publishing House, 2007.

[2] Su Ke. New Concept Font Foundation \& Application. Taibai Literature and Art Press, 2015.

[3] Wang Nanjie, You Hong. Character Design and Originality. Hefei: HeFei University of Technology Press, 2009.

[4] Jackson Tan. Useless Design. Beijing: People's Posts and Telecommunications Press, 2007.

[5] Wang Shaoqiang. Book Forms. China Youth Publishing House, 2012.

[6] Sun I Visual Design. Font Design Rules. Beijing: Publishing House of Electronics Industry, 2012.

[7] Xiao Yong. Character Print Media and Book Design. Hunan: Hunan Fine Arts Publishing House, 2009.

[8] Zhengzhou Municipal Press and Publication Bureau. Ancient Capital Zhengzhou Book Series: Anecdotes. Henan: Elephant Press, 2004.

[9] (UK) Shawn White. Lovely Design. Liaoning Science \& Technology Press, 2009.

[10] Catherine M Fisher. Creative Paper Design. Shanghai: Shanghai People's Fine Arts Publishing House, 2003.

[11] Cai Yushuo, Meng Qinghong. Font Design. Henan: Haiyan Press, 2011.

[12] Xu Nan, Wei Kun. Font Design. Beijing: China Youth Press, 2009. 